\title{
PROTOCOLO DE LAS SESIONES DE TERAPIA INTERPERSONAL APLICADA A UN GRUPO DE FAMILIARES DE PACIENTES CON TRASTORNO DE CONDUCTA ALIMENTARIA
}

\author{
M. LECINA FERNÁNDEZ Y JOSEP SOLÉ \\ Unidad de Salud Mental, Área 11, Gandía (Valencia)
}

(Aceptado en octubre de 2002)

\begin{abstract}
Se presenta un protocolo para aplicar la terapia interpersonal (TIP) a un grupo de familiares de pacientes con trastorno de conducta alimentaria(TCA). Los objetivos de la intervención eran ayudar a los padres a favorecer las relaciones interpersonales con sus hijos con el fin de que mejore a su vez el TCA. Con este fin, se ha aplicado la TIP a un total de 27 familias durante 20 sesiones estructuradas, siguiendo un formato grupal y combinando las estrategias típicas de la TIP con técnicas de tratamiento cognitivo-conductual. La evaluación de la eficacia de la intervención se ha realizado mediante las respuestas a una encuesta semiestructurada que las familias respondieron antes dl tratamiento y a los 12 meses de terminado el mismo. Los resultados indican que se han producido cambios significativos en las relaciones interpersonales familiares como consecuencia del tratamiento.
\end{abstract}

Palabras clave: Terapia interpersonal, trastorno de conducta alimentaria, relaciones familiares, familia, relaciones interpersonales.

\section{A protocol for the application of group interpersonal therapy to relatives of eating disordered patients}

A protocol for the application of the Interpersonal Therapy (IT) to relatives of eating disordered (ED) patients is presented. The aim of the psychotherapy program was to improve the relationships between the ED patients and their parents in order to favor the cognitive-behavioral treatment of the ED. The IP was applied to 27 families in 20 structured sessions following a group format and combining IP strategies with cognitive-behavioral techniques. The IP efficacy has been assessed using a semi-structured interview (pre-vs. post-treatment, at 12-months follow-up). The results show significant positive changes in the relationships pattern between parents and patients.

Key words: Interpersonal therapy, eating disorders, family relationships, family therapy, interpersonal relationships.

\section{INTRODUCCION}

La Terapia Interpersonal (TIP) fue descrita por Klerman, Weissman, Rounsaville y Chevron, en 1984, como una terapia con un enfoque pluralista, no doctrinario y empírico, construído sobre la experiencia clínica y la evidencia experimental. Es una terapia focal, a corto plazo y de duración limitada, que enfatiza las relaciones interpersonales del paciente aunque reconoce también el papel de los factores

Correspondencia: M. Lecina Fernández, c/ Rio Záncara 20, Urb. Parque Boadilla, 28669 Boadilla del Monte (Madrid). genéticos, bioquímicos, del desarrollo y la personalidad en la génesis de las psicopatologías (fernánde-Liria, Rodríguez, Diéguez, González y Morales, 1997; Lipsitz, Markowitz, Cherry y Fyer, 1999; Schramm, 1998). Klerman et al. (1984) aplicaron la TIP a pacientes depresivos y Fairbun $(1985,1996)$ la aplicó al tratamiento de la bulimia nerviosa.

Los Trastornos de la Conducta Alimentaria (TCA) son considerados en la actualidad desde una perspectiva multidimensional que incluye factores somáticos, psicológicos individuales, familiares y socioculturales, que se supone interac- 
tuan entre sí tanto para desencadenar como para mantener el trastorno (Gardner y Garfinkel 1985, 1996; Morande, 1995; Raich, 1994; Toro y Vilardell,1987; Turón, 1997; Vandereycken y Meerman, 1984;). Partiendo de estas consideraciones, la intervención terapéutica debe incluir además de cambios de conducta y cognitivos, modificaciones sustantivas en las relaciones interpersonales, tanto familiares como sociales.

Son muchos los clínicos que han destacado la importancia de diversos tipos de alteraciones en las relaciones familiares de los pacientes con TCA, así como la dificultad de lograr unos resultados satisfactorios en la intervención psicoterapéutica sin un planteamiento familiar. No es por tanto de extrañar el hecho de que cada vez más la familia ha ido adquiriendo un peso importante dentro de la intervención y el tratamiento de estos trastornos. Así, la familia se ha venido incluyendo desde distintas orientaciones terapéuticas: desde el psicoanálisis, cuyo objetivo era ayudar a las familias a un mejor conocimiento de sus problemas, hasta los modelos estructuralistas - Minuchin y la escuela de Filadelfia, que buscan el cambio de las disfunciones de los patrones transaccionales-acción en lugar de insight-, pasando por el modelo de sistema cibernético familiar (Selvini Palazzoli y la escuela de Milán). La idea común de estas dos últimas escuelas o movimientos de psicoterapia es que la anorexia nerviosa manifiesta alteraciones del sistema familiar, en el que el trastorno cumple un papel «homeostático y estabilizador».

A partir de 1980 aparece la denominada "segunda generación" de terapeutas de familia, que integra elementos de los diferentes modelos y utiliza de forma más flexible y pragmática conceptos y estrategias procedentes de diversas escuelas de terapia familiar. Progresivamente los terapeutas familiares tienden a combinar conceptos y estrategias deriva- dos de diferentes modelos (psicodinámico, conductual, estructural, estratégico, sistémico e interpersonal) para hacer frente a los TCA en programas de tratamiento multidimensional, tanto con pacientes ingresadas como ambulatorias. En todo caso, la consecución de resultados satisfactorios en el tratamiento de esta enfermedad pasa por conseguir la colaboración tanto de la paciente como de la familia, puesto que resulta crucial conseguir un cambio de comportamiento $y$ de actitud hacia la enfermedad por ambas partes.

La estructura y composición de los grupos de padres según los diferentes abordajes terapéuticos toma como modelo el funcionamiento de una familia normal, más que una disfuncional, ya que se piensa que ello permite que todos los miembros participen activamente y se pueden así detectar con más facilidad las áreas conflictivas. La atención se mantiene sobre el aquí y ahora de aspectos que pueden ser abordados como objetivo primario de tratamiento: normalizar la alimentación. Centrarse en la normalidad ayuda a concentrar los esfuerzos del grupo dirigiéndolos hacia una meta.

Los grupos de padres pueden variar en composición y frecuencia, pero en general presentan una serie de ventajas. Diferentes autores (por ej., Vandereycken, Castro y Vardenlinden, 1991), consideran que los grupos de padres son de gran utilidad para lograr una implicación familiar positiva en el tratamiento y ayudar a los padres a enfrentarse con las emociones y problemas provocados por el trastorno del hijo. Según estos autores, los grupos de padres ayudan a aspectos tan diversos como los siguientes: adquirir información sobre la enfermedad, compartir experiencias comunes, reducir los sentimientos de aislamiento, reducir la ansiedad, reducir los sentimientos de culpa, fracaso, aumentar los sentimientos de sentirse entendidos y apoyados, 
aumentar la posibilidad de aprender unos de otros, promover cambios de actitud y conducta.

Teniendo en cuenta por tanto a importancia del papel de la familia y su implicación en el tratamiento, este trabajo presenta un programa de intervención con Terapia Interpersonal, adaptada a un grupo de familiares de enfermos de TCA siguiendo los planteamientos de Klerman et al. (1984)

Los bjetivos del programa fueron los siguientes:

1. Ayudar a las familias a favorecer las relaciones interpersonales padreshijos con el fin de que mejore, a su vez, el TCA.

2. Que las familias sean capaces de cambiar la actitud hacia la paciente, a partir del conocimiento de la enfermedad y de la conducta problema.

3. Ganar una razonable independencia por ambas partes (familia y paciente), que favorezca nuevas conductas más adaptativas.

La intervención estuvo dirigida a familiares de enfermos de TCA pertenecientes a la Unidad de Salud Mental de Gandia, Area 11 de la Consellería de Sanitat de la Generalitat Valenciana, que acudían en busca de ayuda y/o tratamiento para sus hijos, derivados de Atención Primaria o de otros servicios. Hasta el momento se ha atendido a un total de 27 familias, distribuidas en seis grupos. En este trabajo se expone la evolución del estudio del primer grupo sobre el que se realizó la intervención, ya que es el único del que hasta el momento se dispone de datos relativos al seguimiento (1 año). El esquema que se seguirá es el siguiente: en primer lugar, se comentan las características de los participantes (familiares y pacientes), para pasar a describir a continuación los principales resultados obtenidos y las características genéricas del programa terapéutico. Después se especifican los contenidos del programa según cada una de las fases y sus respectivas sesiones.

\section{PARTICIPANTES}

La selección de la muestra se llevó a cabo por muestreo consecutivo durante el tercer trimestre de 1997. Los criterios de selección, para la inclusión en el grupo de familiares de enfermos de TCA, fueron los siguientes: (1) Características de enfermedad o exposición: familiares de enfermos de TCA, incluyendo los diagnósticos de anorexia nerviosa y bulimia nerviosa; (2) Características de accesibilidad: asistencia con regularidad a las sesiones; (3) Historia de tratamientos: Todos los sujetos podían haber recibido tratamientos anteriores (tanto los familiares como los enfermos), pero en el caso de los familiares, debía ser ésta la primera intervención de tipo familiar que recibían.

\section{Características de las familias}

El número total de participantes ha sido de ocho personas. Todas eran mujeres, madres de las pacientes con TCA, con una edad media de 48 años. Sus niveles socio-económico y cultural eran medio-bajo y la mayoría (90\%) eran amas de casa.

De ellas, dos abandonaron el tratamiento. Ambas presentaban también bulimia nerviosa con 4 y 5 años de evolución respectivamente, además de características de personalidad límite. Una tenía historia de varios abandonos de distintos tratamientos anteriores y la otra caso no había recibido, ni recibe, tratamiento alguno. En ambos casos había una historia de importantes conflictos de relación entre los progenitores. 


\section{Características de las pacientes}

Las ocho pacientes, todas ellas mujeres, tenían un diagnóstico de TCA, seguían tratamiento individual ambulatorio psicológico con una orientación cognitivo-conductual y haciendo énfasis en la mejora de la autoestima y resolución de problemas. Ninguna de ellas presentaba un peso inferior al $15 \%$ de lo que le correspondía, pero si los restantes criterios de diagnóstico de anorexia nerviosa o bulimia nerviosa según el DSM-IV. No habían estado hospitalizadas por TCA, aunque si habían recibido otros tratamientos. Las edades de los pacientes oscilaban entre 16-29 años y eran estudiantes de BUP, FP, o COU, si bien dos de ellos estaban ya incorporados al mundo laboral.

\section{Protocolo de Evaluación}

Se aplicó una encuesta a cada familiar al iniciar y finalizar cada una de las sesiones. Era semiestructurada y de respuesta abierta, con el objetivo de evaluar el estado de las relaciones interpersonales y emociones expresadas hacia los hijos según cinco componentes: comentarios críticos sobre la paciente, sentimientos negativos hacia la paciente, consecuencias emocionales, afecto, y comentarios positivos hacia la paciente.

\section{RESULTADOS DE LA INTERVENCIÓN}

En la Tabla 1 se exponen los principales resultados derivados de la encuesta que sirvió como protocolo de evaluación, tal y como se describe en el apartado precedente. Como puede constarse, las diferencias entre el pre-tratamiento y el seguimiento a un año (post), son especialmente alentadores, especialmente teniendo en cuenta el bajo nivel cultural de los familiares participantes y la complejidad del problema. La tasa de comentarios críticos negativos y de sentimientos asimismo negativos y disfuncionales, disminuyó de manera significativa. Por su parte, los aspectos positivos de la relación, se incrementaron de forma notable.

De todos modos, somos conscientes de que estos resultados solo pueden considerarse como provisionales, y que harían falta protocolos de evaluación más fiables y estructurados para llegar a conclusiones razonables.

\section{DESARROLLO DEL PROGRAMA: PLANTEAMIENTO GENERAL}

\section{Duración del tratamiento}

Las sesiones de grupo se han llevado a cabo durante un tiempo de ocho meses con una duración de unos setenta minutos. Los primeros cuatro meses, las sesiones tuvieron una frecuencia semanal y

Tabla 1. Porcentajes de respuesta pre y post intervención de terapia interpersonal en familiares de pacientes con trastornos de conducta alimentaria

\begin{tabular}{lcc}
\hline & Pre-intervención & Post-intervención \\
\hline Comentarios críticos negativos hacia el paciente sobre sus formas & & 2 \\
$\quad$ de relación & 8,5 & 1,2 \\
Sentimientos negativos hacia el paciente & 8 & 2,5 \\
Sentimientos de tristeza y preocupación & 2 & 0 \\
Sentimientos de culpabilidad & 2,2 & $\mathbf{7 , 5}$ \\
Comunicación de afecto hacia el paciente & 2 & 8,5 \\
Comentarios positivos hacia el paciente & & \\
\hline
\end{tabular}


los siguientes cuatro quincenal. Las revisiones de seguimiento se han realizado cada dos meses, durante un año.

\section{Características del trabajo en grupo}

Las características generales del trabajo llevado a cabo en el grupo eran las siguientes:

- Entrega material escrito.

- Propuesta de tareas para casa.

- Revisión de autorregistros.

- Apoyo de documentación (casos publicados, artículos, vídeo).

- Utilización de una pizarra para ejemplificar los problemas y sus posibles soluciones.

\section{Estructuración de las Sesiones}

Las sesiones se estructuraron siguiendo el diseño habitual de la TIP en tres niveles:

1) nivel de las estrategias para llevar a cabo determinadas tareas,

2) nivel de las técnicas, $y$

3) nivel del estilo terapéutico.

De estos tres niveles, el relativo a las estrategias es la aportación más específica de la TIP y se emplean en las tres fases del tratamiento. En la primera fase, se identifica el principal problema asociado al inicio del trastorno alimentario y se realiza un contrato con la familia para trabajar esta área-problema. En la fase intermedia se trabajan las principales áreas problema interpersonales actuales. En la fase de terminación se habla de la finalización, de los progresos efectuados y del trabajo que resta por hacer. A continuación, se describe el protocolo utilizado en las diferentes fases del tratamiento.

\section{Protocolo de las sesiones de TIP}

Además de la sesión previa de presentación, el programa se estructura como se ha dicho en tres fases: una Inicial (con 7 sesiones), otra Intermedia (9) y la de Finalización (4 sesiones). El contenido de cada una de estas sesiones es el que sigue.

I. Presentación: El objetivo es reunir a los familiares y explicar a todos juntos y al mismo tiempo los objetivos y contenidos del trabajo en grupo. Se entrega la encuesta a cada uno de los familiares y se aclaran las dudas que puedan surgir al respecto. Los objetivos específicos son por tanto: (1) presentación del grupo, (2) explicar objetivos y contenidos, y (3) entrega y explicación de la encuesta.

II. Fase primera. Incluía las siguientes sesiones:

Sesión 1. Las madres describen lo que les ha motivado a buscar tratamiento, la historia reciente de su condición como madres de un/a hijo/a con TCA y una revisión de las síntomas que presentan. El objetivo de esta sesión se define como: "ocuparse del problema de familiares de personas con TCA». Los objetivos específicos son:

- Revisar los síntomas de familias de TCA: Se revisan los síntomas en las familias según la bibliografía y se aclara que no hay una tipología familiar única.

- Dar un nombre al síndrome: «Padres afectados»: Informar que no se ha considerado a los padres ni como enfermos, ni como terapeutas. Desde la perspectiva de la TIP se ha considerado como más adecuado el concepto de "padres afectados"

- Explicar el TCA Se informa de la seriedad del trastorno, tanto desde el punto de vista de los riesgos físicos, como de la naturaleza psicológica del mismo.

- Otorgar el rol de enfermo al paciente con TCA

- Recogida de la encuesta. 
Sesión 2. El objetivo genérico de esta sesión consiste en relacionar el TCA. con el contexto interpersonal. Para ello, se propone:

- Revisar las relaciones interpersonales presentes y pasadas y relacionarlas con los síntomas de TCA

- Examinar con la familia la naturaleza de la interacción de sus relaciones interpersonales con personas significativas (especialmente con el hijo/a con TCA). El objetivo es que las familias informen de cómo son sus relaciones interpersonales con personas allegadas a ellas y que las comparen con las que tienen con sus hijas/os y si la relación ha cambiado a medida que ha ido avanzando el TCA

Sesión 3. En esta sesión se pretende seguir examinado las relaciones del TCA con el contexto interpersonal. Para ello, se propone:

- Analizar las expectativas del familiar hacia su hijo/a y si están siendo satisfechas. El objetivo es ayudar a las familias a clarificar cuales han sido en el tiempo las expectativas hacia sus hijas y cuales son las expectativas actuales en su TCA y si han sido y están siendo satisfechas.

Sesión 4. En esta sesión se continúan examinado las relaciones del TCA con el contexto interpersonal. El énfasis se sitúa aquí en:

- Analizar los aspectos satisfactorios e insatisfactorios de las relaciones con los/as hijos/as con TCA. El objetivo es determinar con las familias qué aspectos consideran en la relación interpersonal con sus hijas como relaciones satisfactorias, qué acontecimientos en sus relaciones recuerdan como agradables y los sentimientos que les producen. A continuación, se hace lo mismo con los aspectos insatisfactorios.

- Se plantea el tema de la sesión siguiente y se pide que traigan por escrito los cambios que desearían en la relación con su hija.

Sesión 5. El objetivo sigue siendo poner en relación el TCA con el contexto interpersonal. En este caso, se abordan concretamente:

- Los cambios que el familiar desearía en sus relaciones con su hijo/a enfermo. El objetivo es ayudar a las familias a clarificar los cambios que desearía en las relaciones con sus hijas y ayudarles a focalizar el cambio de sus relaciones interpersonales hacia una dirección más amplia y satisfactoria ya que prácticamente su relación está centrada en el TCA
Sesión 6. El objetivo general de esta sesión es el de identificar las áreas más problemáticas de las relaciones con los pacientes. Con este fin, se determina cuál de entre las cuatro áreas problema que describe la TIP convencional (Duelo, Disputas interpersonales, Cambio de rol y Déficits interpersonales) es la que se va a elegir para trabajar en las sesiones. El área de las disputas constituye el aspecto más destacado del TCA, por lo que se tiende a insistir en esta como elección principal. Su elección permite a su vez ayudar a las familias a fijar los objetivos de la terapia, teniendo en cuenta que estén relacionados con el problema principal: el TCA. La delimitación concreta de los contenidos de la sesión es el siguiente:

- Determinar el área de problemas más relacionada con el TCA y fijar los objetivos del tratamiento.

- Determinar que aspecto de la relación está relacionado con el TCA y sería óptimo cambiar.

- Tarea para casa: cada familiar debe escribir los objetivos que se propone, para conseguir su propio cambio.

Sesión 7. El objetivo terapéutico se circunscribe a la formalización del contrato, esto es, el compromiso verbal de la familia a asistir a las sesiones del grupo. Los contenidos específicos se concretan del modo siguiente:

- Fijar objetivos (2 6 3): El familiar define además para cada uno de los objetivos cual sería el mejor resultado posible, el más probable y el peor.

- Utilizar este proceso como feedback.

III. Fase Intermedia: Disputas interpersonales. Hacen referencia a las situaciones en las que el familiar afectado y su hijo/a enfermo tienen expectativas no recíprocas sobre su relación. La TIP considera este tipo de discrepancias como especialmente importantes en la génesis y perpetuación de la depresión: por lo tanto, en este caso se trata de su valoración como importantes en la perpetuación de los TCA Las sesiones de esta fase están centradas en este área problema y el objetivo general es identificar la disputa, escoger un plan de acción y modificar las expectativas y la comunicación para conseguir una solución 
satisfactoria. La estrategia consiste en ayudar a los familiares a identificar la disputa, a relacionar los síntomas del/a paciente con la disputa, y a entender cómo las expectativas no recíprocas se relacionan con la disputa. Se exploran los motivos de la disputa, las diferentes expectativas y valores, qué se desearía de la relación, qué opciones se tienen, y de qué recursos se dispone para cambiar la relación.

Sesión 1. El plan de acción se dirige a:

- Modificar los patrones de comunicación. Se introducen técnicas de comunicación cognitivo-conductuales: estilos de comunicación, entrenamiento en elogios y refuerzos positivos dirigidos hacia su persona, aspecto físico y habilidades.

- Reevaluación de las expectativas.

- Se inicia la información sobre técnicas para la resolución de conflictos. En nuestro caso se ha introducido la filosofía de resolución de problemas, siguiendo los pasos de Terapia de Resolución de Problemas de D'Zurrilla y Golfried, relacionándolo en todo momento con las estrategias de la TIP.

- Tareas para casa: Registro de tres elogios diarios hacia la hija haciendo referencia a sus cualidades físicas, personales y/o habilidades, expresando sentimientos personales, con el objetivo de ayudar a suavizar el estilo de comunicación en la relación y ayudar a fortalecer y profundizar la relación interpersonal .

Sesión 2. El objetivo de esta sesión es observar la dificultad de la tarea de elogiar y expresar sentimientos positivos hacia la persona de su hija, asentar las bases de la técnica aprendida y corregir y mejorar su aplicación. Por ello, se propone:

- Modificar patrones de comunicación.

- Revisión de las tareas de elogios.

Sesión 3. Pretende esclarecer los objetivos y relacionarlos con las disputas. Centramos los objetivos en el cambio y mejora de la relación interpersonal (madre-hija-padre) y no en el cambio y cura de la enfermedad de la hija directa e inmediatamente. Es decir, los objetivos en este momento no se centran en el cambio de personalidad o cambio inminente de ali- mentación, sino en la mejora de la relación interpersonal.

- Revisión de elogios

- Teoría de la comunicación.

- Componentes de la comunicación.

Sesión 4. El objetivo de esta sesión es informar de los distintos estilos de comunicación: el estilo pasivo, el estilo agresivo, el estilo asertivo y el pasivo-agresivo. Y que el familiar a medida que los aprende intente identificar cuál es el estilo que suele utilizar en sus relaciones interpersonales y concretamente con su hija. Las acciones concretas consisten en:

- Revisar las tareas

- Revisión de componentes de la comunicación.

- Estilos de comunicación.

- Ejercicios de estilo de comunicación durante la sesión.

- Ejercicios de papel y lápiz para clasificar los estilos.

- Tareas para casa: Observar los estilos de comunicación en sus respectivas relaciones familiares.

Sesión 5. El objetivo es confirmar si los familiares saben identificar su estilo de comunicación en sus relaciones interpersonales y en concreto con la paciente, observar si éste tiene relación con las disputas y, si es así, aprender a modificar su patrón de comunicación, para facilitar la relación. Los componentes concretos son:

- Revisar Estilos de comunicación.

- Trabajar con ejemplos personales.

- Modificar el patrón de comunicación.

- Relacionar los estilos de comunicación con las disputas

- Y ayudar a identificar las disputas.

- Comentario de un ejemplo de un caso del libro "Aprende a comer" (Schmidt y Treasure, 1996) para observar y evaluar los problemas claves del caso y la importancia de la comunicación y la mejora de ella y el papel de los Refuerzos positivos en la comunicación.

- Reevaluar expectativas.

Sesión 6. El objetivo de esta sesión es el de reevaluar expectativas e integrar en esta tarea todo lo aprendido hasta ahora en las sesiones previas, desde el concepto de enfermo hasta su estilo de interacción en la relación. Observar qué patrones han cambiado y cuáles faltaría modificar.

- Recordar el concepto de enfermedad-enfermo. 
- Situaciones en las que el familiar afectado y su hijo/a con TCA. tienen expectativas no recíprocas sobre su relación.

- Tarea para casa: Autorregistro de situaciones de disputa con diferentes expectativas.

A partir de esta sesión, las reuniones se realizan quincenalmente.

Sesión 7. El objetivo es ayudar a identificar la disputa y los antecedentes de la misma. Identificar los temas de discusión y relacionarlos con las expectativas no recíprocas y hacer énfasis en aumentar los temas de comunicación que estrechan y satisfacen la relación. Se pretende ayudar al familiar afectado a entender cómo las expectativas no recíprocas se relacionan con las disputas e iniciar los pasos que pueden conducir a la resolución de las disputas y la negociación de roles. Los contenidos específicos son:

- Repasar la sesión anterior.

- Comentar los resultados de la encuesta sobre los items 1 y 2 (Comentarios críticos sobre la paciente y Sentimientos negativos hacia la paciente) y relacionarlos con la sesión.

- Siguiendo con las habilidades de comunicación se introduce la técnica de cómo hacer críticas.

- Tareas para casa:

- Comunicar con lenguaje no absolutista.

- Reforzar las cualidades del hijo/a y conversar sobre temas que le interesen

- Practicar la extinción con los problemas de conducta relacionados con la comida

Sesión 8. El objetivo es dar a las familias información sobre la alimentación y los alimentos, dando la importancia que merece este tema y que observen que la mejora de la relación con sus respectivas hijas no ha estado en función de tener al alcance esta información. Para ello se procede a:

- Revisiar las tareas.

- Partimos de la tarea de la sesión anterior y en relación a la extinción con la comida y a las discusiones que provoca, hablamos por primera vez de los alimentos.

- Mitos e ideas irracionales sobre los alimentos.

- Tabla de composición de alimentos.

- Aportes recomendados de sustancias nutritivas.

Sesión 9. El objetivo es mantener el nivel de relación alcanzado hasta aquí y afianzarlo reforzando todos sus progresos. Adicionalmente, se aporta la Técnica de Resolución de Pro- blemas para ayudar a manejarse ante situaciones problema de la vida cotidiana similares a los de cualquier familia. Los contenidos concretos son:

- Repasar los temas que interesan o destacan más en la relación con la hija. Seguir el esquema de la sesión previa a la anterior.

- Repasar los temas de discusión de la mencionada sesión.

- Introducimos la filosofía de resolución de problemas de D'Zurrilla y Goldfried con un ejemplo ajeno a las situaciones problema que estamos tratando.

Como en la próxima sesión se inician las sesiones de terminación, pasamos un cuestionario para evaluar hasta qué punto está el familiar aprendiendo a manejar el problema, cuáles son sus recursos actuales, y si cabe sugerir alguna corrección o alternativas para lograr relaciones mas satisfactorias.

IV. Fase de terminación. Las 4 últimas sesiones se desarrollan con una frecuencia quincenal. Se recuerda a los participantes la inminencia de la terminación del grupo con el fin de motivarles a aprovechar al máximo estas sesiones que quedan y terminar de alcanzar los objetivos propuestos. Se reconoce y valora la capacidad de aprendizaje de los familiares participantes respecto al manejo del problema y por tanto su capacidad de independencia. Una estrategia útil puede consistir en comparar con ellas los resultados de los cuestionarios de evaluación. Se trabajan las técnicas de resolución de problemas y cómo hacer críticas para la resolución de conflictos relacionándolas con las estrategias de TIP que se han visto en las sesiones anteriores.

\section{Sesión 1. Tema central: conflictos}

- Definición del problema

a. ¿cuál es el problema que nos encontramos?

b. ¿coincidimos todos los familiares implicados en la misma definición de problema? ¿Tenemos en cuenta las expectativas de unos y otros?. 
- Alternativas, Pros y contras de cada una, Elección de la más adecuada, Puesta en marcha y Evaluación.

- Técnicas de comunicación aplicables:

- Escucha activa

- Dar importancia a cada idea y persona que la expone.

- Hablar despacio, tranquilo y suave.

- No agobiar con el tiempo, tomarse el tiempo necesario.

Sesión 2. El objetivo es seguir evaluando si ha habido discusiones o problemas y cual ha sido el modo de afrontarlos y resolverlos. Valorar si los padres han cambiado el patrón de comunicación, y en concreto revisar los ejercicios de resolución de problemas. Contenidos:

- Repasar e insistir en la resolución de problemas de la sesión anterior.

- Evaluar cómo se resuelven los problemas y, si es correcto, reforzar.

Sesión 3. El objetivo es seguir promoviendo cambios en los patrones de comunicación, $y$ concretamente en la forma de hacer y recibir críticas al identificarlas como desencadenante de una discusión sobre la comida o formas de ser cuando no coinciden las expectativas, observación que hicimos en las sesiones iniciales. Por tanto, se trata de relacionar las expectativas con la conducta a criticar. Además, se recuerda a los participantes que es la penúltima sesión y que una vez terminada esta fase, se realizaran únicamente sesiones de seguimiento bimensuales.

Las estrategias que se ponen en práctica siguen este esquema:

Cómo hacer una crítica:

- Describir.: Ejemplo: «Cuando........

- Efectos: a) Efecto objetivo: describir lo que sucede; b) Efecto subjetivo: describir cómo me siento

- Responsabilizarse del problema

- Entender al otro: Ejemplo:»Entiendo que es difícil....

- Buscar alternativas. Ejemplo: «Me gustaría que....» «Lo más adecuado sería que......»

- Reforzar el cambio.

- Avisar de las consecuencias: Advertir de lo que el sujeto se pierde (cosas positivas) por no hacer

- Relacionar las expectativas con la conducta a criticar.

Sesión 4. El objetivo fundamental es alentar a las familias a que funcionen por ellas mismas res- pecto al manejo del problema tal como lo están haciendo actualmente y mantengan en la práctica lo aprendido en las sesiones. Los contenidos se concretan así:

- Repasar sesión anterior.

- Despedida hasta la sesión de seguimiento dentro de dos meses.

- Entrega de un cuadernillo de ejercicios a los familiares.

\section{Fase de Seguimiento. Las sesiones} de seguimiento se realizan cada dos meses durante un periodo de seis meses. En caso de no acudir el familiar a las sesiones, se mantiene contacto telefónico. Es conveniente mantener un seguimiento de la familia durante un periodo de un año más con entrevista o telefónicamente. Durante todo este tiempo, las enfermas de TCA continúan con su tratamiento, si es el caso.

\section{CONCLUSIONES}

La familia no es desde luego el único factor causal en el desarrollo de los TCA y está por demostrar que los conflictos en la familia, previos al desarrollo del trastorno, sean la causa de esta enfermedad. En este trabajo no se ha pretendido estudiar la relación causaefecto que pudiera darse entre un familiar significativo (la madre) y la aparición del TCA, sino que interesaba saber si esta intervención, especialmente diseñada por los autores para familiares de pacientes con TCA, era capaz de producir una mejoría en el estilo de vida de las familias afectadas.

A lo largo de nuestra experiencia clínica hemos observado reiteradamente que en las familias con miembros que padecen TCA las relaciones interpersonales se van focalizando en la enfermedad, los patrones de comunicación se van reduciendo al tema de la alimentación, a las formas de comer, a la dieta 
elegida, a la cantidad y calidad de los alimentos ingeridos, a las críticas sobre el mal aspecto físico que va adquiriendo la enferma, y todo ello, unido a la negación de enfermedad por parte del paciente y la preocupación por parte de los padres, va provocando unos estilos de comunicación agresivos, de competitividad entre padres e hijos, en donde las discusiones son tan habituales que llegan a convertirse en el pilar de las relaciones. Se crea un ambiente de conflicto casi permanente, disputas continuas que no suelen alcanzar el objetivo de cambiar la conducta del paciente y mucho menos su actitud, sino que provocan por lo general más disputas, más insatisfacciones, con lo que se favorece la aparición y continuación de los síntomas, creándose un círculo vicioso difícil de resolver.

Si no se encuentra solución el problema permanece o se agrava y las relaciones interpersonales se convierten en un factor más de perpetuación, sin olvidar que otros posibles factores, como los sociales e individuales y las propias características de la enfermedad, siguen influyendo.

Este trabajo ha intervenido directamente en el cambio de las relaciones interpersonales en la familia. Se ha dado prioridad al cambio de las relaciones interpersonales, anteponiéndolas a la creencia por parte de los padres de la necesidad de saber única o fundamentalmente cómo deben ser sus reacciones ante las conductas restrictivas y/o bulímicas de las hijas.

La estrategia se ha centrado en desfocalizar su atención de la conducta alimentaria y centrarla más en mejorar sus relaciones, amoldándose a expectativas más realistas, cambiar los patrones de comunicación, aplicar las técnicas adecuadas para aumentar la frecuencia e intensidad de las relaciones satisfactorias, expresar sentimientos positivos, etc.
Los resultados positivos obtenidos hasta el momento corroboran la importancia de realizar una intervención familiar en el tratamiento de los TCA, y pensamos que justifican nuestra propuesta de continuar trabajando en esta misma línea, combinado los principios de la TIP con los propios de las terapias cognitivo-comportamentales.

\section{REFERENCIAS BIBLIOGRÁFICAS}

Fairburn,C.G. (1985). Cognitive-behavioral treatment for bulimia. En: Garner, D.M. y Garfinkel, P.E (Eds): Handbook of psychotherapy of anorexia and bulimia. NuevaYork: Guilford Press.

Fairburn, C.G. (1996). Interpersonal Psychotherapy for bulimia nervosa. En Garner, D.M. and Garfinkel P.E. (Eds.). Handbook of treatment of eating disorders. Nueva York. Guilford Press.

Fernández Liria, A., Rodriguez, B., Dieguez, M., González, N., y Morales, N. (1997). La terapia interpersonal de la depresión y las psicoterapias en el fin del milenio. Revista de la Asociación Española de Neuropsiquiatría, 17, 627 648.

Garner, D.M. y Garfinkel, P.E (Eds): Handbook of psychotherapy of anorexia and bulimia. NuevaYork: Guilford Press.

Garner, D.M. and Garfinkel P.E. (Eds.) Handbook of treatment of eating disorders. Nueva York. Guilford Press.

Lipsitz, J.D., Markowitz, J.C., Cherry, S., y Fyer, A.J. (1999). Open study on interpersonal theraphy for the treatment of social phobia American Journal of Psychiatry, 156, 1814-1816.

Klerman, G.L., Weissman, M.M., Rounsaville, B.J. y Chevron, E.S. (1984). Interpersonal psychotherapy of depression. Nueva York: Basic Books.

Morande, G. (1995) Un peligro llamado anorexia. La tentación de adelgazar. Barcelona: Temas de hoy.

Raich, R.M. (1994). Anorexia y bulimia: trastornos de alimentación. Madrid: Pirámide. 
Schmidt,U. y Treasure, J. (1996). Aprender a comer. Barcelona: Martinez Roca.

Schramm, E. (1998). Psicoterapia Interpersonal. Barcelona, Masson.

Toro, J. y Vilardell, E. (1987). Anorexia nerviosa. Barcelona, Martinez Roca.
Turón, V.J. (1997). Trastornos de la alimentación. Anorexia nerviosa, bulimia y obesidad. Barcelona, Masson.

Vandereycken,W., Castro,J. y Vanderlinden, J. (1991). Anorexia y bulimia. La familia en su génesis y tratamieento. Barcelona, Martinez Roca. 